\title{
Identification of a gene expression signature associated with the metastasis suppressor function of NME1: prognostic value in human melanoma
}

\author{
M Kathryn Leonard ${ }^{1,2,6}$, Joseph R McCorkle 3,4,6,7, Devin E Snyder ${ }^{1,2,6}$, Marian Novak ${ }^{1,2,7,8}$, Qingbei Zhang ${ }^{1,2,7}$, \\ Amol C Shetty ${ }^{5}$, Anup A Mahurkar ${ }^{5}$ and David M Kaetzel ${ }^{1,2}$
}

Although NME1 is well known for its ability to suppress metastasis of melanoma, the molecular mechanisms underlying this activity are not completely understood. Herein, we utilized a bioinformatics approach to systematically identify genes whose expression is correlated with the metastasis suppressor function of NME1. This was accomplished through a search for genes that were regulated by NME1, but not by NME1 variants lacking metastasis suppressor activity. This approach identified a number of novel genes, such as ALDOC, CXCL11, LRP1b, and XAGE1 as well as known targets such as NETO2, which were collectively designated as an NME1-Regulated Metastasis Suppressor Signature (MSS). The MSS was associated with prolonged overall survival in a large cohort of melanoma patients in The Cancer Genome Atlas (TCGA). The median overall survival of melanoma patients with elevated expression of the MSS genes was > 5.6 years longer compared with that of patients with lower expression of the MSS genes. These data demonstrate that NMEl represents a powerful tool for identifying genes whose expression is associated with metastasis and survival of melanoma patients, suggesting their potential applications as prognostic markers and therapeutic targets in advanced forms of this lethal cancer.

Laboratory Investigation (2018) 98, 327-338; doi:10.1038/labinvest.2017.108; published online 23 October 2017

Melanoma is the most lethal form of skin cancer, with an enhanced ability to metastasize to distant organs via the hematogenous or lymphatic circulation. ${ }^{1}$ Although recently developed inhibitors of MAPK pathway components and immune checkpoint mediators represent the first meaningful progress in the treatment of advanced forms of melanoma, ${ }^{2,3}$ most patients still develop resistance to these agents and succumb to metastatic disease. Improved prognostic markers and therapeutic targets are acutely needed to optimize therapy and achieve durable responses in patients with advanced melanoma.

Metastasis suppressor genes (MSGs) encode both proteins ${ }^{4,5}$ and miRNAs ${ }^{6}$ with the unique ability to selectively inhibit metastatic potential of tumor cells, with little effect on growth of the primary tumor. As such, MSGs could represent powerful experimental tools for unraveling molecular mechanisms that specifically regulate the metastatic process. The NME1 gene (also known as NM23-H1 or NDPK-A) was the first MSG identified, by virtue of its diminished expression in a metastatic melanoma cell line of murine origin. ${ }^{7}$ Reduced NME1 expression has since been correlated with metastatic forms of melanoma ${ }^{8}$ and many other cancers. ${ }^{9}$ In addition, forced expression of NME1 suppresses metastatic potential of numerous cancer cell lines both in cell culture and as xenografts in immunocompromised mice. ${ }^{10,11}$ We recently showed that concomitant ablation of the NME1 and NME2 genes greatly enhances metastatic potential in a mouse model of ultraviolet (UV) light-induced melanoma, ${ }^{12}$

\footnotetext{
'Department of Biochemistry and Molecular Biology, School of Medicine, University of Maryland-Baltimore, Baltimore, MD, USA; ${ }^{2}$ Marlene and Stewart Greenebaum Comprehensive Cancer Center, University of Maryland-Baltimore, Baltimore, MD, USA; ${ }^{3}$ Hematological Malignancies Program, St Jude Children's Research Hospital, Memphis, TN, USA; ${ }^{4}$ Department of Pharmaceutical Sciences, St Jude Children's Research Hospital, Memphis, TN, USA and ${ }^{5}$ Institute for Genome Sciences, School of Medicine, University of Maryland-Baltimore, Baltimore, MD, USA

Correspondence: DM Kaetzel, PhD, Department of Biochemistry and Molecular Biology, School of Medicine, University of Maryland-Baltimore, 108 North Greene Street, Baltimore, MD 21201, USA.

E-mail: DKaetzel@som.umaryland.edu

${ }^{6}$ These authors contributed equally to this work.

${ }^{7}$ This work was performed as part of the dissertation studies of Drs McCorkle, Novak, and Zhang in the Department of Molecular and Biomedical Pharmacology, University of Kentucky, Lexington, KY 40536-0298, USA.

${ }^{8}$ Current Address: Personal Genome Diagnostics, 2809 Boston Street \#503, Baltimore, MD 21224, USA.

Received 21 May 2017; revised 19 July 2017; accepted 25 July 2017
} 
providing strong in vivo evidence of its metastasis suppressor function. Multiple reports suggest that the NME1 protein regulates tumor cell motility via physical and functional interactions with a variety of signaling pathways. ${ }^{13}$ However, NME1 has also been reported to control RNA expression profiles in breast carcinoma ${ }^{14}$ and melanoma cell lines, ${ }^{15}$ suggesting that this activity could also mediate its metastasis suppressor function.

NME1 is a member of a 10-gene family (NME1-9 and $R P 2)$, with four of the encoded NME proteins exhibiting nucleoside diphosphate kinase (NDPK) activity. ${ }^{16}$ The NDPK activity is well recognized for its role in balancing of intracellular pools of nucleotide diphosphates and triphosphates. ${ }^{17}$ NME1 is recruited to protein complexes within the cytoskeleton and other intracellular compartments, where the NDPK function may transfer NTPs directly to effector proteins through a process termed 'substrate channeling. ${ }^{18}$ A role for NDPK activity in the metastasis suppressor function of NME1 is yet to be firmly established, however, with at least one study suggesting that it does not contribute. ${ }^{19}$ Nonetheless, relevance of NDPK is strongly suggested in other settings by the direct association of NME1/ NDPK protein with a variety of cytoskeletal and membrane mediators of cell motility. ${ }^{13}$ In addition, NDPK activity has been shown to supply GTP to dynamin during membrane remodeling and endocytosis of cell surface receptors, ${ }^{20}$ a function that could well influence metastatic potential of cancer cells. Moreover, disruption of the NDPK-active site by a site-directed point mutation of the catalytic histidine-118 residue $\left(\mathrm{H}_{118} \mathrm{~F}\right)$ modestly impaired metastasis suppressor function of NME1 in the human melanoma cell line 1205Lu. ${ }^{11}$ NME1 also binds single-stranded DNA, ${ }^{21,22}$ suggesting roles in transcription and DNA repair. ${ }^{23}$ Indeed, expression of NME1 and NME2 promotes nucleotide excision repair of UV-induced lesions, ${ }^{24}$ while NME1/2 deficiency renders mice prone to $\mathrm{UV}$-induced melanoma in situ. ${ }^{25}$ Moreover, NME1 exhibits $3{ }^{\prime}-5^{\prime}$ exonuclease activity toward single-stranded DNA templates in vitro, consistent with roles in DNA proofreading and/or repair. ${ }^{26}$ Site-directed mutagenesis of NME1 and NME2 has identified a number of aminoacid residues critical for enzymatic and metastasis suppressor functions. ${ }^{27-29}$ Our site-directed mutagenesis analysis of the NME1 molecule revealed amino-acid residues $\mathrm{E}_{5}$ and $\mathrm{K}_{12}$ to be essential for both $3^{\prime}-5^{\prime}$ exonuclease activity (ie, DNA repair) and the metastasis suppressor function, ${ }^{11}$ a correlation that suggests NME1 may suppress metastasis-driving mutations in melanoma and other human cancers.

NME1 also regulates expression of genes that could serve as effectors of its metastasis suppressor activity. We posited that genes that were regulated by wild-type (WT) NME1, but not the suppressor-deficient mutants $\mathrm{E}_{5} \mathrm{~A}$ and $\mathrm{K}_{12} \mathrm{Q}$, would yield a signature enriched with genes that mediate metastasis suppressor activity. The approach involved forced expression of WT NME1 or one of the metastasis suppressor-deficient mutants in the human melanoma cell line, WM793, followed by assessment of their impacts on gene expression by microarray analysis. The approach identified multiple genes that alone and cumulatively are predictors of improved survival in patients with melanoma. This NME1-dependent metastasis suppressor signature (MSS) contains a number of genes with potential as prognostic markers and targets for therapy in advanced melanoma.

\section{MATERIALS AND METHODS Cell Lines and Culture Conditions}

The melanoma cell lines WM793 and WM278 were gifts of Dr Meenhard Herlyn (Wistar Institute, Philadelphia, PA, USA). WM793 and WM278 cells were cultured at $37^{\circ} \mathrm{C}$ and $5 \% \mathrm{CO}_{2}$ in $\mathrm{Tu} 2 \%$ media, composed of the following: MCDB: Leibovitz-15 medium (4:1, v/v; Sigma-Aldrich, St Louis, MO, USA) supplemented with $2 \mathrm{mM} \mathrm{CaCl}_{2}, 2.5 \mu \mathrm{g} / \mathrm{ml}$ insulin, and 2\% fetal bovine serum (Life Technologies, Grand Island, NY, USA). MDA-MB-435s/M14 cells (referred to as M14 in this text) were obtained from R. Plattner (University of Kentucky). ${ }^{30}$ 293t cells were obtained from the American Type Culture Collection (Manassas, VA, USA). M14 and 293t cells were maintained at $37^{\circ} \mathrm{C}$ and $10 \% \quad \mathrm{CO}_{2}$ in DMEM supplemented with $10 \%$ fetal bovine serum, $4.5 \mathrm{~g} / \mathrm{l} \mathrm{D}$-glucose, L-glutamine, and $110 \mathrm{mg} / \mathrm{l}$ sodium pyruvate. Wild-type and mutant variants of NME1 were prepared and characterized as described previously. ${ }^{11}$ Briefly, cDNA of WT or mutant variants of NME1 was cloned into mammalian expression vector pCI-EGFP (provided by S Kraner, University of Kentucky), followed by transfection into WM793 or M14 cells. Stable transfectants were selected by 2 week exposure to G418, followed by enrichment for GFP-positive cells by cell sorting. Stable knockdown of NME1 expression in WM278 cells was achieved by lentiviral delivery of Mission shRNA (Sigma-Aldrich) TRCN0000010062 (shNME1\#1) or Nontarget shRNA pLK0.1 (SHC002, Sigma-Aldrich) as a negative control. Production of viral vectors and transduction were performed according to the manufacturer's instructions.

\section{Experimental Metastasis}

Six-to-seven-week-old female nude mice (nu/nu, Harlan Laboratories, Indianapolis, IN, USA) were injected intravenously with $2 \times 10^{6}$ cells in a total volume of $100 \mu \mathrm{l}$ Hank's Buffered Saline (Invitrogen, Grand Island, NY, USA). The mice were killed 3 weeks later and the lungs were promptly removed, rinsed in PBS, and immersed in Bouin's fixative (Polysciences, Warrington, PA, USA) to preserve the tissue and enhance the visibility of potential metastatic lesions. The lesions were counted and their diameter was measured using a dissecting microscope.

\section{RNA Isolation and Microarray Analysis}

Parental WM793 cells or WM793 stably expressing WT or mutant NME1 were seeded at a density of $1.5 \times 10^{5}$ per $100-$ $\mathrm{mm}$ plastic dish and were grown for 3 days to $75-80 \%$ confluence in $\mathrm{Tu} 2 \%$. Total cellular RNA was harvested 
(RNeasy Extraction Kit, Qiagen, Valencia, CA, USA) from five independent dishes for each cell line and treatment group. Purified RNA samples were diluted to $1 \mathrm{mg} / \mathrm{ml}$ with RNAse-free deionized water and transferred to the University of Kentucky Microarray Core Facility for cDNA synthesis, hybridization, and scanning using their established protocols (http://www.research.uky.edu/microarray/AffymetrixGeneChip. $\mathrm{html}$ ). Genome-wide expression profiling was conducted using GeneChip ${ }^{\circledR}$ Human Exon 1.0 ST Arrays (Affymetrix, Santa Clara, CA, USA). Data analysis of the microarrays was conducted by Informatics Resources Center within the Institute for Genome Sciences at the University of Maryland-Baltimore (Baltimore, MD, USA). Briefly, arrays were normalized by the Robust Multi-Array Average method ${ }^{31}$ implemented in $\mathrm{R}$ package. Differential expression analysis was performed with Linear Models of Microarrays (LIMMA) R package. A linear model was first fitted to expression data for each gene, and the empirical Bayes method was then used to assess differential expression between two WT NME1 cells and metastasis suppressor-deficient cell lines (parent WM793, $\mathrm{E}_{5} \mathrm{~A}$, or $\mathrm{K}_{12} \mathrm{Q}$ NME1 mutant lines). A $P$-value cutoff of less than 0.01 was used to select significant probes. Microarray data from this study are deposited in the Gene Enrichment Omnibus under accession number GSE85978.

\section{Enrichment Analysis}

Enrichment analysis was conducted using DAVID Bioinformatics Resources 6.8 Beta $^{32,33}$ and QIAGEN's Ingenuity ${ }^{\circledR}$ Pathway Analysis (IPA ${ }^{\circledR}$, QIAGEN Redwood City, www. qiagen.com/ingenuity). Exon array probe sets differentially expressed following NME1 wild-type overexpression in WM793 cells $(P<0.05)$ were analyzed against the Gene Ontology Consortium (GO) database and Ingenuity Knowledge Base using all Human Exon 1.0 ST probe sets as background. Statistical significance was determined following multiple testing correction (ie, Bonferroni and BenjaminiHochberg).

\section{Nanostring Analysis of RNA Expression}

WM793, WM278, and M14 cells, and their respective stably transfected variants, were plated at $1.5 \times 10^{5}$ per $100-\mathrm{mm}$ plastic dish and were grown for 3 days to $75-80 \%$ confluence. Cells were then serum-starved for $24 \mathrm{~h}$ before harvesting total cellular RNA (RNeasy Extraction Kit, Qiagen) from three independent dishes. For Nanostring transcriptome analysis a custom plate measuring 16 genes of interest was conducted using $250 \mathrm{ng}$ of total RNA $(50 \mathrm{ng} / \mu \mathrm{l})$ per sample. Five additional housekeeping genes were measured and used for normalization. A complete list of gene targets and probe sequences can be found in Supplementary Table 1. Data analysis was conducted using the nSolver (v.2.5) user interface to operate the nCounter Advanced analysis module from Nanostring (Nanostring Technologies, Seattle, WA, USA). Genes of interest were only considered detectable if the mean of the normalized signal was greater than or equal to two s.d.'s above the mean of the negative controls.

\section{TCGA Analyses}

The Cancer Genome Atlas (TCGA) mRNA expression data, from Skin Cutaneous Melanoma (SKCM) samples, was obtained through GDAC FireHose (v01-28-2016). The results shown here are in whole or part based upon data generated by the TCGA Research Network: http://cancergenome.nih.gov/. The acquired data set was run through the TCGA IlluminaHiSeq_RNASeqV2 pipeline. The mRNA expression data were imported into RStudio and were normalized using the voom function found in the LIMMA package (v1.0.136, RStudio, Boston, MA, USA). The resulting normalized expression values were converted into $z$-scores and, subsequently, expression profiles for specific genes of interest were generated and analyzed using heatmap.2 function found in the gplots package.

Clinical information for TCGA SKCM patients was also obtained through FireHose version 01-28-2016 and imported into RStudio. In order to determine a relationship between gene expression and clinical outcome, SKCM patient identifiers from both clinical and $z$-score information were matched. Patients were grouped based on their gene expression profile and analyzed for alterations in both overall survival and recurrence-free survival. ${ }^{34}$ Kaplan-Meier survival analysis was completed through the survival package in $\mathrm{R}$.

\section{RESULTS \\ NME1 Regulates the Profile of RNA Expression in WM793 Melanoma Cells}

To analyze the impact of NME1 on RNA expression profiles in melanoma, a cDNA containing the NME1-coding sequence without $5^{\prime}$ - and $3^{\prime}$-untranslated sequences was expressed by stable transfection in the human melanoma cell line WM793. The parental WM793 line was derived from a vertical growth phase (VGP) melanoma and exhibits invasive characteristics in culture, ${ }^{35,36}$ as well as very low endogenous expression of NME1 protein. ${ }^{11}$ For RNA expression analysis, total RNA was isolated from WM793 cells and the stably transfected, WM793-derived line expressing NME1 protein at $\sim 3-4 \times$ endogenous levels. ${ }^{11}$ Cells were subjected to $24 \mathrm{~h}$ of serum starvation before RNA preparation to minimize induction of gene transcription by serum-associated growth factors. RNA samples were analyzed by microarray using the Affymetrix Human Exon 1.0 ST platform, which assesses expression for 21014 gene products and variants.

A comparison of expression profiles from the two WM793derived cell lines identified a total of 153 RNAs whose expression was significantly altered $(P<0.05)$ by NME1 (Supplementary Table 2). Filtering of the data at higher stringency $(P<0.01)$ yielded 21 RNAs differentially regulated by NME1, 14 of which were upregulated and seven downregulated (Figure 1a). Gene ontology analysis identified that half of the genes fell into three functional groupings that were 

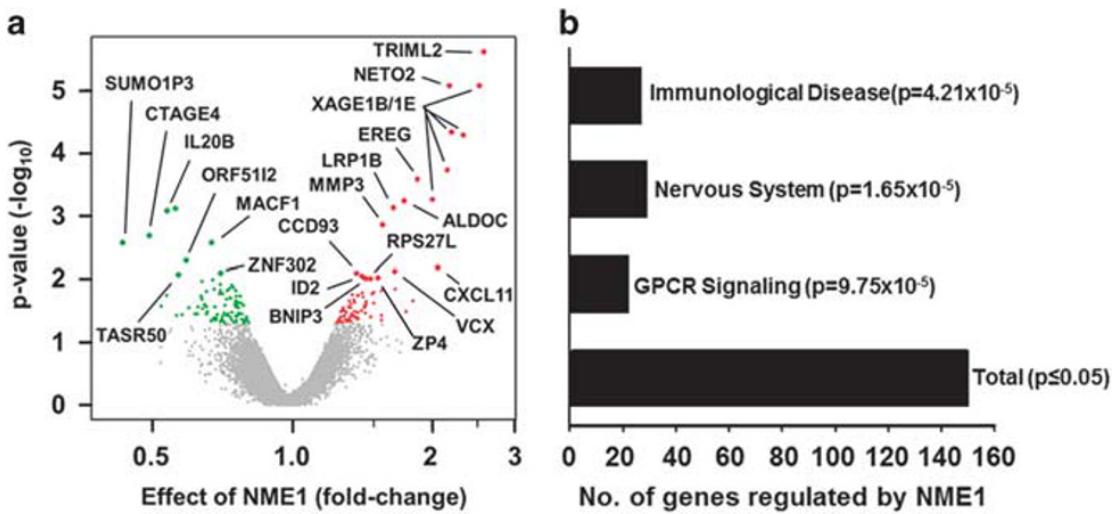

Figure 1 Microarray analysis of genes differentially expressed (DE) in WM793 cells after stable forced expression of NME1. (a) Volcano plot representation of RNA expression changes following forced NME1 expression in WM793 melanoma cells. Closed red circles depict transcripts upregulated by forced NME1 expression $(P<0.05)$, with green closed circles indicating downregulated transcripts. Transcripts not significantly regulated by NME1 are shown in grey. Transcripts most robustly influenced by NME1 $(P<0.01)$ are shown as larger closed circles and are labeled with their corresponding gene ID. (b) Shown is the number of DE genes significantly enriched in various pathways, as determined by gene ontology in DAVID and Ingenuity Pathway Analysis (IPA) analyses.

significantly enriched in the list of NME1-regulated genes, namely immunological disease, nervous system development and function, and GPCR signaling (Figure $1 \mathrm{~b}$ ). The remaining NME1-regulated genes that did not belong to these three ontological groups were associated with ontological groups that failed to achieve statistical significance in the analysis.

\section{Impact of NME1 and Point Mutant Variants on Experimental Metastasis of WM793 Cells}

Although NME1 regulates expression of gene networks in cancer-derived cell lines, the impacts of this activity on its metastasis suppressor function are not fully understood. ${ }^{14,15}$ To identify specific genes and networks mediating metastasis suppressor function of NME1 in melanoma, a filtering strategy was devised to detect genes regulated by the WT NME1 protein, but not regulated by mutant variants of NME1 we have shown previously to lack metastasis suppressor activity. For the current study, a panel of WM793-derived cell lines was used that had been created by stable transfection to provide forced expression of WT NME1 and the NME1 point mutants glutamate-5 to alanine $\left(\mathrm{E}_{5} \mathrm{~A}\right)$, lysine-12 to glutamine $\left(\mathrm{K}_{12} \mathrm{Q}\right)$, and histidine-118 to phenylalanine $\left(\mathrm{H}_{118} \mathrm{~F}\right)$. The $\mathrm{E}_{5} \mathrm{~A}$ variant is deficient in $3^{\prime}-5^{\prime}$ exonuclease activity, variant $\mathrm{K}_{12} \mathrm{Q}$ is deficient in both the $3^{\prime}-5^{\prime}$ exonuclease and NDPK activities, and $\mathrm{H}_{118} \mathrm{~F}$ is deficient only in the NDPK activity. Of these point mutants, $\mathrm{E}_{5} \mathrm{~A}$ and $\mathrm{K}_{12} \mathrm{Q}$ are deficient in metastasis suppressor activity in context of the human melanoma cell line 1205Lu. ${ }^{11}$ NME1 is rarely mutated in cancers; however, the use of point mutations that fail to suppress metastasis allows for more streamlined examination of the metastasis suppressor functions of NME1 in experimental settings. The WM793 panel was considered for the current study in light of its higher levels of forced NME1 expression than obtained in the $1205 \mathrm{Lu}$ panel, with each NME1 mutant variant expressed at approximately fourfold over-endogenous NME1 levels (Figure 2a). ${ }^{11}$ Before RNA expression analysis, metastatic potential of the WM793 panel was measured by experimental metastasis assays, performed by injection of cells into the tail vein of athymic nude mice. Significant lung colonization by WM793 cells was seen in $60 \%$ of injected mice, whereas forced expression of WT NME1 strongly suppressed this activity as expected $(8.7 \%$; Table 1). In contrast, expression of the $\mathrm{E}_{5} \mathrm{~A}$ or $\mathrm{K}_{12} \mathrm{Q}$ variants had no significant suppressor effect on lung-colonizing activity (50\% lung colony-positive for both), consistent with loss of metastasis suppressor activity, as previously described in $1205 \mathrm{Lu}$ cells. ${ }^{11}$ Also consistent with prior results in $1205 \mathrm{Lu}$ cells, ${ }^{11}$ expression of the NDPK-deficient variant $\mathrm{H}_{118} \mathrm{~F}$ had no statistically significant effect on lung colonization in the WM793 line. Taken together, these analyses showed the WM793-derived cell lines expressing WT NME1 and the $\mathrm{E}_{5} \mathrm{~A}$ and $\mathrm{K}_{12} \mathrm{Q}$ variants would provide a robust system for identifying genes whose expression tracks with the metastasis suppressor function of NME1.

\section{Identification of a MSS of RNA Expression in Human Melanoma Cell Lines}

To better identify which of the 153 genes regulated by WT NME1 were associated with its metastasis suppressor activity, microarray analyses were also conducted in WM793 cells expressing the metastasis suppressor-deficient mutants of NME1 $\left(\mathrm{E}_{5} \mathrm{~A}\right.$ and $\left.\mathrm{K}_{12} \mathrm{Q}\right)$. The marked loss of metastasis suppressor activity for the $\mathrm{E}_{5} \mathrm{~A}$ and $\mathrm{K}_{12} \mathrm{Q}$ mutants made them ideal tools for identifying NME1-regulated genes mediating the suppressor function. It should be noted that the NME1 point mutations used in our filtering approach were generated in vitro for the purpose of dissecting structure-function relationships for the NME1 molecule and are not known to occur naturally within the NME1 sequence in melanoma or other forms of human cancer. The $\mathrm{H}_{118} \mathrm{~F}$-dependent gene 
a

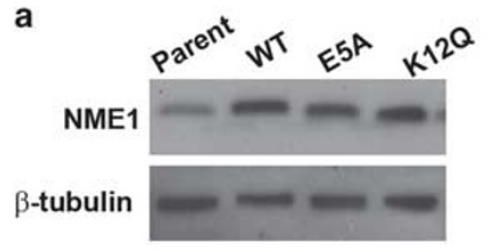

b

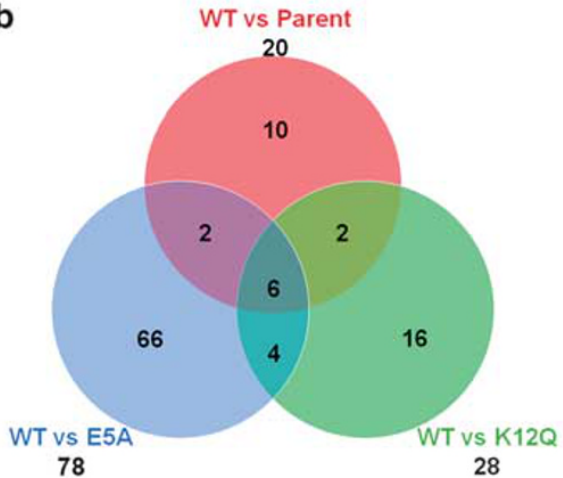

C

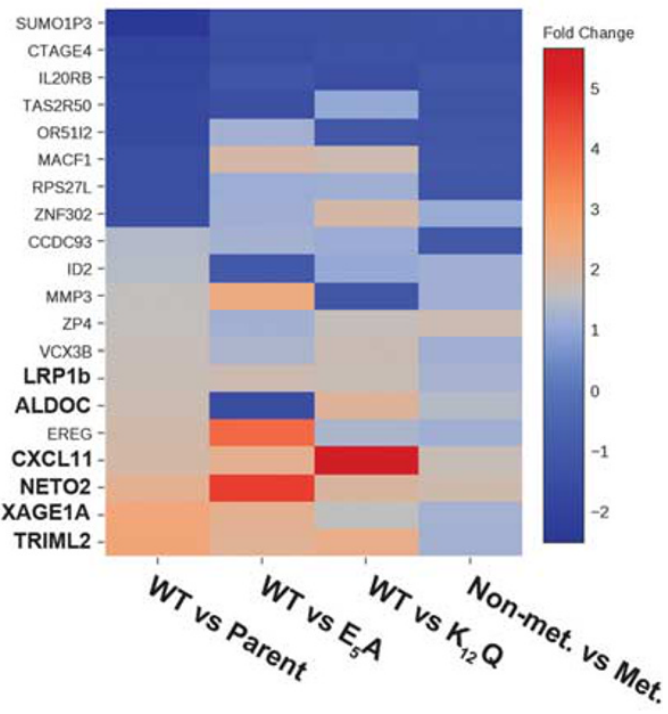

Figure 2 Identification of NME1-regulated genes that track with metastasis suppressor activity of NME1. (a) Validation of wild-type (WT) or mutant NME1 overexpresssion in WM793 cells by immunoblot analysis. (b) Venn diagram depicting the overlap of genes differentially expressed by WT vs metastasis suppressor-deficient mutants of NME1 or parent cells, $P=0.01$. (c) Heatmap showing the relative induction or repression of NME1-regulated genes in all comparisons of cell lines. The six genes common to all comparisons from (b) are shown in bold text.

Table 1 Amino-acid residues glutamate-5 and lysine-12 mediate ability of NME1 to suppress experimental metastasis potential of WM793 melanoma cells

\begin{tabular}{lccccc}
\hline $\begin{array}{l}\text { Cell lines; exogenous } \\
\text { NME1 }\end{array}$ & \multicolumn{2}{c}{$\begin{array}{c}\text { NME1 enzymatic } \\
\text { activity }\end{array}$} & & \multicolumn{2}{c}{ Experimental metastasis } \\
\cline { 2 - 3 } & NDPK & $3^{\prime}-5^{\prime}$ EXO & & $\%$ (Incidence) & Lesions/lung \\
\hline & - & - & & $60^{\mathrm{a}}(12 / 20)$ & $6^{\mathrm{a}}$ \\
Wild-type & + & + & & $8.7^{\mathrm{b}}(2 / 23)$ & $4^{\mathrm{a}, \mathrm{b}}$ \\
$\mathrm{E}_{5} \mathrm{~A}$ & + & - & & $50^{\mathrm{a}, \mathrm{c}}(5 / 10)$ & $1^{\mathrm{a}, \mathrm{b}, \mathrm{c}}$ \\
$\mathrm{K}_{12} \mathrm{Q}$ & - & - & & $50^{\mathrm{a}, \mathrm{c}}(5 / 10)$ & $2^{\mathrm{a}, \mathrm{d}}$ \\
$\mathrm{H}_{118} \mathrm{~F}$ & + & - & & $25^{\mathrm{a}, \mathrm{b}, \mathrm{c}}(3 / 12)$ & $2^{\mathrm{a}, \mathrm{b}, \mathrm{e}}$ \\
\hline
\end{tabular}

EXO, exonuclease; $\mathrm{H}_{118} \mathrm{~F}$, histidine-to-phenylalanine; $\mathrm{E}_{5} \mathrm{~A}$, glutamate-toalanine substitution; $\mathrm{K}_{12} \mathrm{Q}$, lysine-to-glutamine; NDPK, nucleoside diphosphate kinase.

Values not showing a common superscript are significantly different $(P<0.05)$ as determined by Fisher's exact test (incidence) and Student's $t$-test (lesions/lung).

+ , in vitro enzymatic activity of recombinant NME1 variant $>95 \%$ of wild-type NME1.

- , in vitro enzymatic activity of recombinant NME1 variant $<5 \%$ of wild-type NME1.

expression profile was not included in our filtering approach for the MSS, as the $\mathrm{H}_{118} \mathrm{~F}$ mutation did not significantly alter the metastasis suppressor activity of NME1 in vivo (Table 1). After three separate comparisons, (WT vs Parent, WT $v s \mathrm{E}_{5} \mathrm{~A}$, and $\mathrm{WT} v s \mathrm{~K}_{12} \mathrm{Q}$ ) were compiled, six genes were consistently regulated by WT NME1 but not by a metastasis suppressordeficient mutant (Figures $2 \mathrm{~b}$ and $\mathrm{c}$ ). This gene expression signature consisted of LDL receptor-related protein ( $L R P 1 b)$, C-X-C motif chemokine 11 (CXCL11), aldolase C (ALDOC), $\mathrm{X}$ antigen family member $1 \mathrm{~A}(X A G E 1 A)$, neuropilin and tolloid-like 2 (NETO2), and tripartite motif family like 2 (TRIML2). For this report, these genes have been designated as the NME1-regulated MSS. To validate results obtained by microarray analysis, Nanostring analysis was employed for quantitation of NME1 and the following NME1-regulated transcripts: ALDOC, CXCL11, EREG, LRP1b, NETO2, and TRIML2. In addition, we measured expression of MSS genes in $\mathrm{H}_{118} \mathrm{~F}$-expressing WM793 cells to identify any potential contributions of the NDPK function to their regulation. In agreement with microarray and immunoblot analyses, the Nanostring approach demonstrated a three- to fourfold induction of NME1 RNA in WM793 cell lines expressing WT NME1 and the variants $\mathrm{E}_{5} \mathrm{~A}, \mathrm{~K}_{12} \mathrm{Q}$, and $\mathrm{H}_{118} \mathrm{~F}$ (Figure 3a). Consistent with the microarray analysis, Nanostring validated the induction of EREG, LRP1b, and TRIML2 transcripts by WT NME1, and not the metastasis suppressordeficient variants $\mathrm{E}_{5} \mathrm{~A}$ and $\mathrm{K}_{12} \mathrm{Q}$. Moreover, similar to the microarray analyses, Nanostring results indicated that NME1mediated induction of ALDOC mRNA was dependent on the NDPK function. WT and $\mathrm{E}_{5} \mathrm{~A}$ forms of NME1, which both retain full NDPK activity, induced ALDOC mRNA but the $\mathrm{K}_{12} \mathrm{Q}$ and $\mathrm{H}_{118} \mathrm{~F}$ mutants did not. The Nanostring analysis yielded considerable variability with impacts of the $\mathrm{K}_{12} \mathrm{Q}$ mutant on expression of the CXCL11 and NETO2 transcripts limiting the ability to interpret if one or more enzymatic 

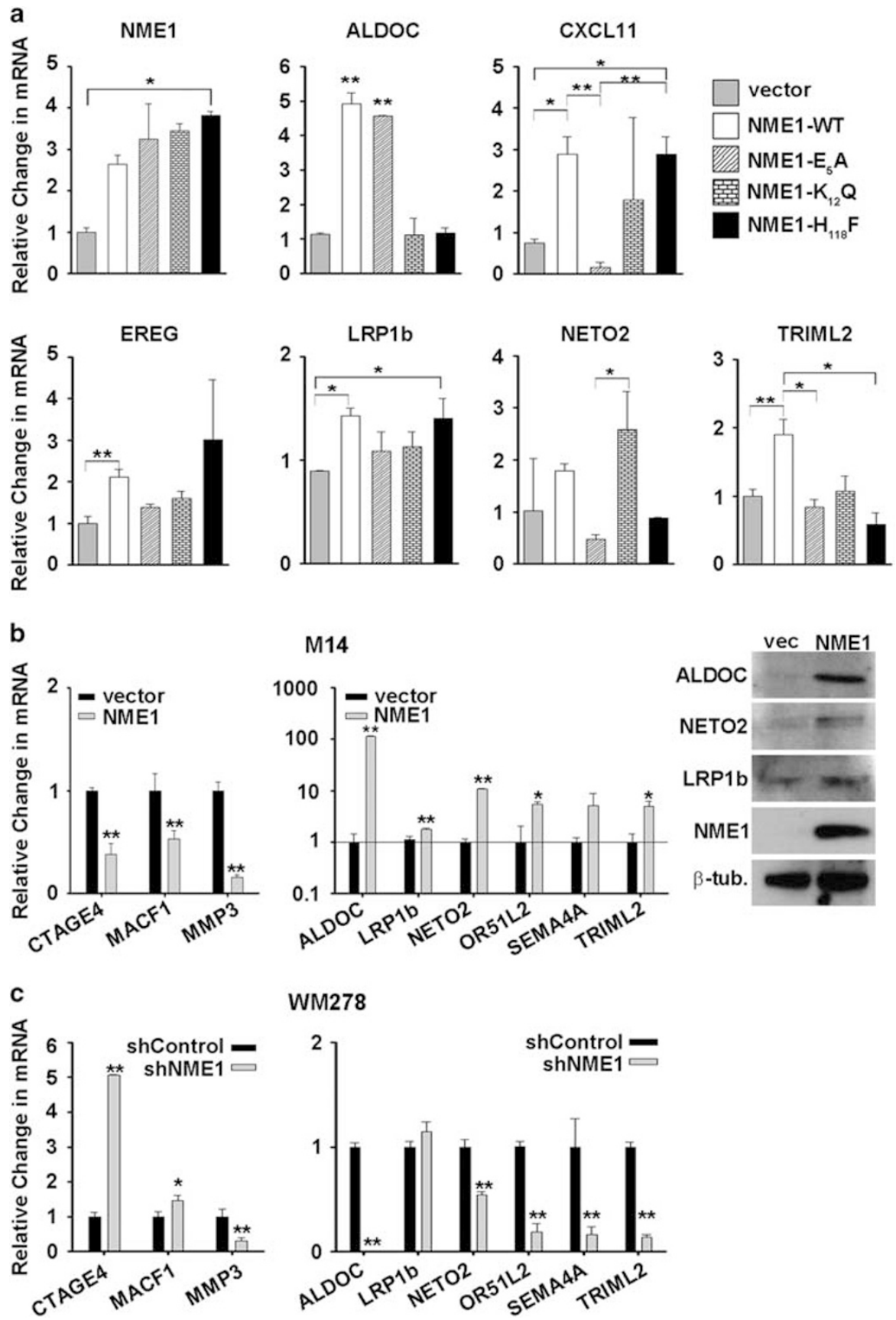

WM278

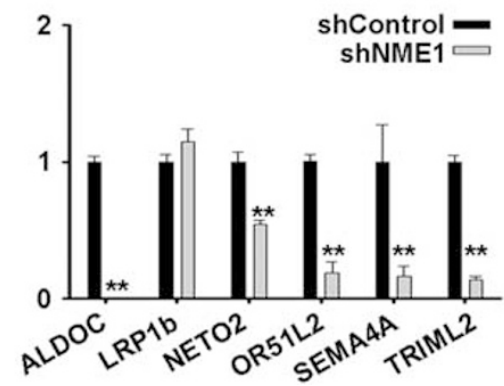

Figure 3 NME1-regulated genes show variable dependence on NME1 enzymatic functions and cell line expression. (a) Nanostring-mediated validation of target gene levels after stable expression of empty vector (gray bars), wild-type (WT), NDPK activity-deficient NME1 mutants $\left(\mathrm{K}_{12} \mathrm{Q}\right.$ and $\left.\mathrm{H}_{118} \mathrm{~F}\right)$, or $3^{\prime}-5^{\prime}$ exonuclease-deficient mutants $\left(\mathrm{E}_{5} \mathrm{~A}\right.$ and $\mathrm{K}_{12} \mathrm{Q}$ ). (b) The repression or induction of select NME1 target genes after stable expression of wild-type NME1 was further verified in M14 cells by Nanostring (left panel) and immunoblot analyses. (c) The effect of NME1 knockdown on its target genes was measured by Nanostring analysis in WM278 cells after stable expression of a control or NME1-specific shRNA. ${ }^{*} P<0.05,{ }^{* *} P<0.01$.

function of NME1 were critical for their expression. On the whole, however, the bulk of the microarray results were validated by the Nanostring approach.

Nanostring analysis was also used to measure levels of all 21 differentially regulated genes after overexpression of WT NME1 in the M14 melanoma cell line. After normalization to several housekeeping genes, $9 / 21$ of those genes were consistently altered in response to NME1, of which four were MSS genes. Three genes that showed repression by microarray analysis in WM793 also showed significant downregulation by WT NME1 in the M14 cells (Figure 3b). Of the NME1-induced genes, the most robust regulation was 
that of $A L D O C$, which was induced over 100-fold (Figure 3b). Other MSS transcripts strongly induced by NME1 in M14 cells were NETO2 (10-fold) and TRIML2 (5-fold), although $L R P 1 b$ was only modestly upregulated in this cell line. Immunoblot analysis confirmed that NME1 overexpression induced steady-state concentrations of ALDOC, NETO2, and LRP1b proteins in M14 cells (Figure 3b).

To further validate NME1-mediated regulation of these genes, the impact of NME1 knockdown was assessed using lentiviral shRNA expression vectors (shNME1) in WM278 cells. This line was originally derived from a VGP melanoma and, in contrast with WM793, expresses relatively high amounts of NME1 protein. As predicted, loss of endogenous NME1 induced the expression of NME1suppressed genes CTAGE4, MACF1, and MMP3 (Figure 3c). Transcript levels of ALDOC, NETO2, OR51L2, SEMA4A, and TRIML2 were strongly reduced by shNME1 in WM278 cells (Figure 3c). Expression of the NME1-induced transcript encoding LRP1b was unaffected by the shNME1 treatment.

Interestingly, each of the MSS genes harbors functions and/ or expression patterns, suggesting relevance to melanoma phenotype and malignant progression. $L R P 1 b$ is a compelling candidate as an effector of the metastasis suppressor function for NME1, with reports of potent invasion-suppressing activity across multiple cancer types including melanoma. ${ }^{37,38}$ In addition, $L R P 1 b$ is one of the top three most-frequently mutated genes in melanoma, consistent with a suppressor function. ${ }^{39} \mathrm{~A}$ closely related protein, LRP1, was recently shown to aid in suppressing melanoma metastasis, further suggesting that LRP1b is critical for NME1-mediated metastasis suppression. ${ }^{40}$ Chemokines such as CXCL11 are key factors in tumor progression and metastasis, acting in both the primary tumor and metastatic niche. The actions of CXCL11 are pleotropic and context-dependent, due in part to autocrine and paracrine expression patterns of its cognate receptors CXCR3 and CXCR7. ${ }^{41}$ Consistent with potential suppressor functions, CXCL11 stimulates recruitment of tumor-infiltrating lymphocytes in T-cell lymphomas ${ }^{42}$ and breast cancer, ${ }^{43}$ whereas its receptor CXCR3 is critical for response to anti-PD-1 therapy in preclinical studies. ${ }^{44}$ ALDOC is one of three aldolase isozymes $(\mathrm{A}-\mathrm{C})$, well recognized for their roles in glycolysis but more recently implicated in novel glycolysis-independent functions in cell proliferation ${ }^{45,46}$ and motility/invasion. ${ }^{47}$ ALDOA interacts physically with ARNO, an ADP-ribosylation factor (Arf) and guanine exchange factor implicated in cytoskeletal dynamics and membrane trafficking. ${ }^{48}$ Consistent with an antimetastatic function, ALDOC expression is correlated with better prognosis in oral squamous cell carcinoma, ${ }^{49}$ whereas the isoenzyme ALDOA is upregulated by melanogenesis-induced cell quiescence in the metastatic melanoma cell line B16F10. ${ }^{50}$ Also relevant is the enriched expression of ALDOC protein in cells of neural crest lineage, from which melanocytes are derived. ${ }^{51,52}$ XAGE1B is localized to nuclear speckles, suggesting a role in RNA processing and/or export, and its expression is strongly elevated in metastatic melanoma. ${ }^{53}$ NETO2 is structurally related to neuropilin, a VEGF receptor and co-receptor for semaphorins, the latter implicated in neural and vascular patterning. NETO2 overexpression has been observed in numerous cancers ${ }^{54}$ and hemangiomas, ${ }^{55}$ and is downregulated in response to NME1 expression in the context of breast cancer. ${ }^{14}$ TRIML2 is a member of the XAGE subfamily/GAGE family of proteins that harbor E3 ubiquitin ligase activity, ${ }^{56}$ a function shown to regulate cancer metastasis. ${ }^{57,58}$

\section{A Subset of NME1-Regulated Genes is Highly Predictive of Metastasis and Reduced Survival in Human Melanoma Patients}

The ability of NME1 to suppress metastatic potential of melanoma cell lines suggests that genes under its regulatory control are associated with metastasis and survival in melanoma patients. To address this possibility, we analyzed expression of NME1-regulated genes within the skin cutaneous melanoma (SCKM) samples of TCGA. Unsupervised hierarchical clustering was performed using the expression of five of the six core MSS genes (LRP1b, CXCL11, ALDOC, NETO2, and TRIML2). An additional target gene, MACF1, was included in the analysis because of the consistent significant changes observed upon manipulation of NME1 expression in multiple cell lines (Figures $3 \mathrm{~b}$ and c). The 468 patients within the SCKM TCGA separated into five general clusters based on expression of the six NME1-regulated genes (Figure 4a). Cluster 5, however, only contained three patients and was excluded from further analysis. Few patients showed drastic changes in expression of any one individual gene (Figure 4a) nor was there substantial predictive power for survival rates with any one gene (data not shown). When combined, however, the six gene signatures were more powerful in predicting overall survival. No significant differences in recurrence-free survival were observed between the individual clusters (Figure 4b). Despite somewhat closer clustering of groups 2 and 3 from the hierarchical clustering, the overall survival of patients in cluster 3 was significantly longer than both clusters 1 and 2 (Figure 4c). Because there were no statistical differences in the overall survival probabilities between clusters 3 and 4, or between 1 and 2, the two pairs of subclusters were pooled for further analyses. Patients in pooled clusters 3-4 had a median survival time of 5.6 years longer than patients in clusters $1-2$ (164.3 vs 96.3 months, $P=0.0002$; Figure $4 \mathrm{~d}$ ). Differences in survival times between the clusters were not due to uneven distribution of primary and metastatic patient samples as there was no significant difference in the total number of patients or percentage of metastatic or primary patients within the groups (Supplementary Table 4). When the expression of each of the six NME1-regulated genes was individually examined, all six were significantly $(P<0.001)$ lower in clusters 1-2 compared with clusters 3-4 (Figure 4e). 


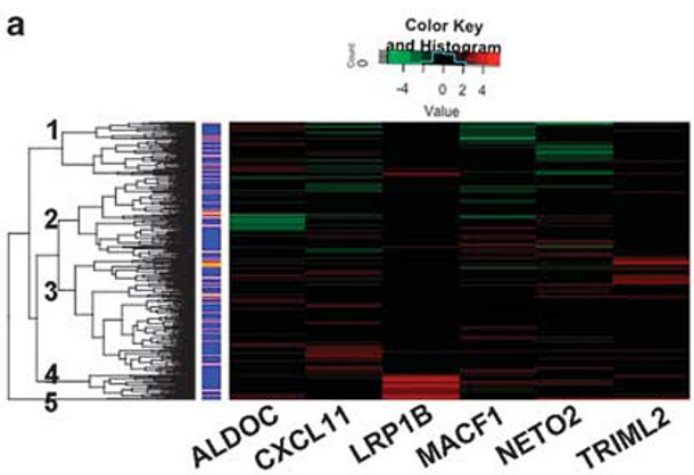

C

\section{Overall Survival}
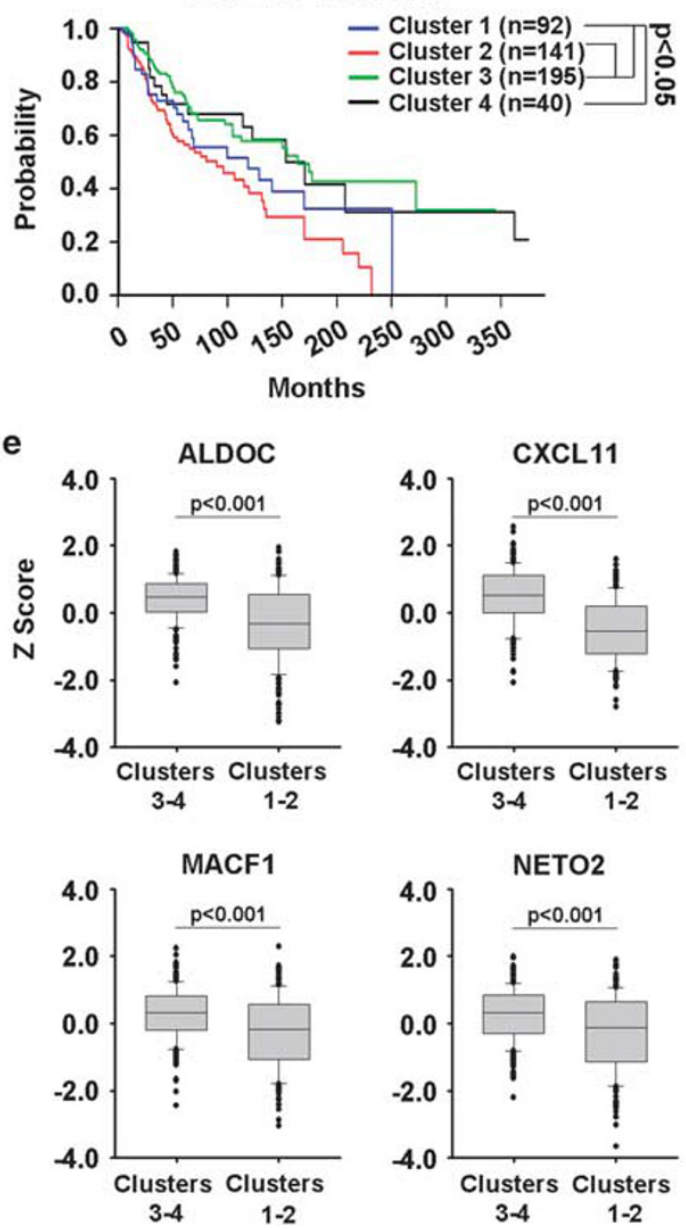

b

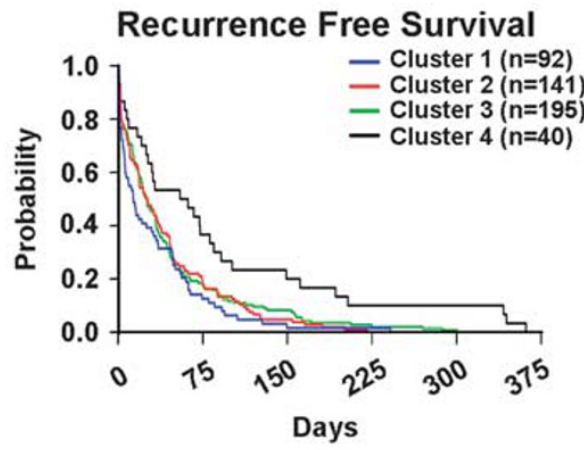

d
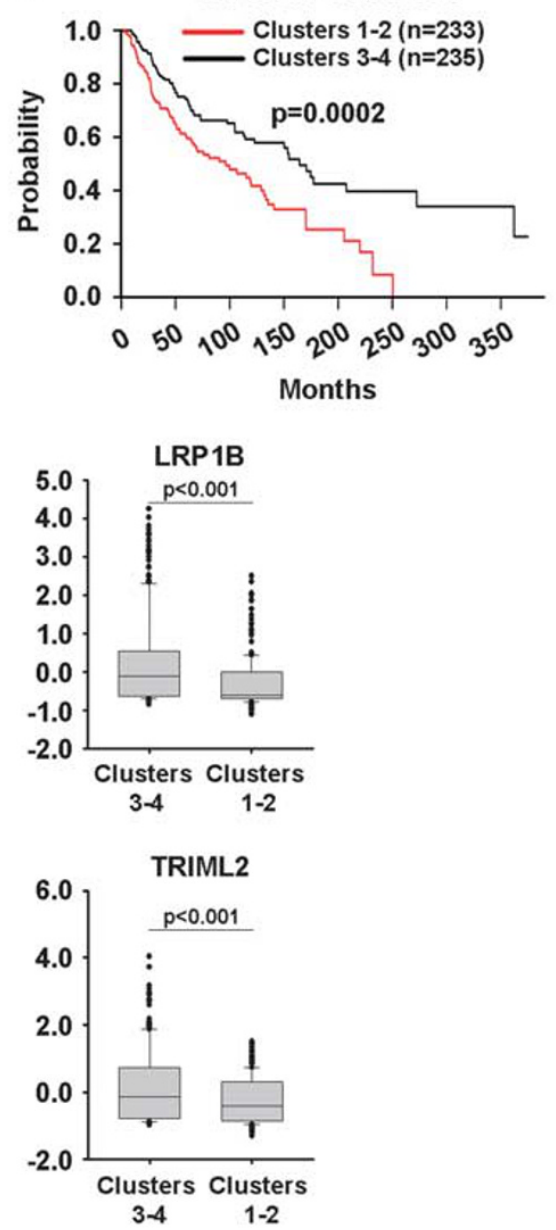

Figure 4 Loss of NME1-induced genes identifies melanoma patients with poor survival. (a) Unsupervised heirarchical clustering based on the expression of NME1-regulated genes in the skin cutaneous melanoma (SKCM) portion of the The Cancer Genome Atlas (TCGA) identifies several clusters. Primary melanomas are designated in orange and metastatic samples in blue. Kaplan-Meier analysis of recurrence-free (b) and overall survival (c) of patients within each cluster. (d) Kaplan-Meier analysis of overall survival after combining cluster 1 with 2, and cluster 3 with 4. (e) RNA expression levels of indicated NME1 target genes within the clusters identified in (a). Stastical differences measured by ANOVA with Holm-Sidak post hoc testing.

Together, these data indicate that reduced expression of these six NME1-regulated genes is associated with poor survival.

Expression of NME1-regulated mRNAs proved to be a stronger predictor of patient outcome than that of the NME1 transcript itself. In fact, after separating patients into the upper and lower quartiles for NME1 expression (Supplementary Figure 1A), elevated NME1 mRNA expression was found to be associated with worse survival in the SCKM TCGA (Supplementary Figure 1B). This counterintuitive observation could be explained by our recent 
demonstration that steady-state concentrations of NME1 mRNA are not the primary determinant of NME1 protein expression in metastatic cells in culture. Instead, NME1 protein is destabilized in metastatic cells via cathepsincatalyzed degradation in the lysosomal compartment. ${ }^{59}$ On the basis of this observation, we predict that RNA/protein expression of the MSS genes would be more strongly correlated with intracellular concentrations of NME1 protein than NME1 mRNA. Unfortunately, the current lack of proteome data in the SCKM-TCGA collection precludes such an analysis. However, expression of 3/6 of the NME1-induced signature genes in the TCGA was inversely correlated with that of NME1 mRNA (Supplementary Figure 1C). This could be explained by an induction of steady-state concentrations of NME1 mRNA levels in response to NME1 protein degradation; reduced expression of MSS genes could represent a response to NME1 protein downregulation, rather than to levels of the NME1 transcript.

Next, the 'low NME1' and 'high NME1' populations were individually subjected to hierarchical cluster analysis of recurrence-free survival, based on expression of the six-gene MSS. The analysis identified four clusters within each population (Supplementary Figure 1D), with all clusters from the low NME1 group exhibiting longer recurrence-free survival than the clusters of the high NME1 group, as expected (Supplementary Figure 1E, left; Supplementary Table 5). Interestingly, cluster 2 from the 'high NME1' group exhibited significantly longer recurrence-free survival than that of cluster 4 of the same 'high NME1' group (Supplementary Figure 1E, right; Supplementary Table 5). No other differences in survival were observed between any clusters within the 'high NME1' group or within the 'low NME1' group. Expression of five of the six MSS genes was significantly different between clusters 2 and 4 (Supplementary Figure 1F). Three MSS transcripts (ALDOC, CXCL11, and LRP1B) were expressed at significantly higher levels in the longer-surviving patients of cluster 2 than in cluster 4. This positive correlation between expression of these MSS transcripts, which are induced by NME1 in melanoma cell lines, and extended survival in melanoma patients is consistent with roles as effectors of the metastasis suppressor function of NME1 in vivo. In addition, two other transcripts (MACF1 and NETO2) were expressed at significantly lower levels in cluster 2 , suggesting that they may be metastasis-driving in nature. Taken together, these analyses demonstrate that expression of the NME1-regulated MSS genes is a strong predictor of prolonged survival in melanoma patients.

\section{DISCUSSION}

Rates of melanoma incidence are steadily increasing, as are death rates from melanoma patients not cured by tumor resection. Even recently developed targeted therapies against the BRAF/MEK signaling axis and immune checkpoint mediators (eg, CTLA-4 and PD-1) have produced only modest improvements in overall survival for most patients with advanced melanoma. ${ }^{60}$ Off-target toxicities and acquired resistance to these agents remain impediments to durable responses. In the short term, clinical management of melanoma patients would be improved greatly by new molecular markers capable of identifying patients likely to progress to more aggressive forms. Such patients would represent the most appropriate candidates for undergoing therapy using these expensive and potentially toxic agents, and possibly for initiation of therapy at earlier stages of their disease. Unfortunately, no reliable markers exist for predicting the course of melanoma, in contrast with the molecular markers currently available for diagnosis and prognosis in breast carcinoma and other cancers. ${ }^{61}$ In the longer term, new therapeutic targets are needed if agents are to be developed with greater efficacy in blocking melanoma progression, and for eradicating metastatic disease once the cancer has progressed. MSGs such as NME1 provide a unique opportunity to identify individual genes and gene networks that are either correlated with metastatic potential (eg, prognostic indices), or are mediators of metastatic activity itself (eg, therapeutic targets). The current study has identified gene networks whose expression is regulated in melanoma cells by the metastasis suppressor NME1, as well as genes that are predictive of better outcome and survival in a large cohort of human melanoma patients.

Ontological analysis identified immunological disease, nervous system development and function, and G proteincoupled receptors (GPCRs) as the three groupings most significantly impacted by forced expression of NME1 in WM793 melanoma cells (Supplementary Table 3). Regulation of genes involved in immune system function was robust (18.1\% of significant genes) and is interesting in light of the critical role of immune cells in elimination of tumor cells in melanoma and other cancers, ${ }^{62}$ as well as the importance of immune cells in the metastatic niche. ${ }^{63}$ Of particular potential interest in this category is the presence of three chemokine/ chemokine receptor genes (CXCL11, CCL8, and CCR2) and the interleukin-20 receptor beta. Genes identified in this functional grouping would seem worthy of further scrutiny as prognostic indicators for responsiveness to inhibitors of immune checkpoint molecules (eg, PD-1 and CTLA-4), which are currently inadequate. The observed enrichment of genes involved in nervous system development and function is also intriguing in light of the neural crest origin of melanocytes, and the phenotypic similarities exhibited by neural crest stem cells and melanoma cells. ${ }^{64}$ Considering the close relationship proposed between stemness and metastatic potential in cancer cells, ${ }^{65}$ the genes identified in this category may represent rational drug targets for metastatic disease in melanoma and other cancers. GPCRs are becoming better recognized for their roles as key regulators of tumor and metastatic phenotypes, especially in melanoma. ${ }^{66}$ GPCRs are frequently mutated in melanoma and other cancers, ${ }^{67}$ exemplified by the identification of GRIN2A mutations in 
over $33 \%$ of human melanomas. ${ }^{68}$ GPCRs of particular interest were 11 olfactory receptor genes suppressed by NME1 in WM793 cells (Supplementary Table 3). Ectopic expression of olfactory receptor genes and proliferation-inhibitory activity has been described previously in melanocytes, ${ }^{69}$ an intriguing observation suggesting potential impact of NME1 on cellular proliferation and differentiation in melanoma.

Our study utilized NME1 variants that we showed previously to be deficient not only in metastasis suppressor activity, but also in NDPK and $3^{\prime}-5^{\prime}$ exonuclease activities of the NME1 protein. Although NME1 is rarely mutated in cancers, these variants represent powerful experimental tools to help differentiate the metastasis suppressor functions of NME1 from other potential housekeeping functions. In the current study as well as our prior one, ${ }^{11}$ metastasis suppressor function was disrupted significantly only in those variants lacking $3^{\prime}-5^{\prime}$ exonuclease activity $\left(\mathrm{E}_{5} \mathrm{~A}\right.$ and $\left.\mathrm{K}_{12} \mathrm{Q}\right)$, suggesting the possibility that this enzymatic function contributes in some way to regulation of the MSS genes. Whereas a molecular mechanism underlying this regulatory function is yet to be described, the preference of the $3^{\prime}-5^{\prime}$ exonuclease for single-stranded DNA substrates ${ }^{22,26}$ suggests a role in remodeling of regulatory elements in DNA that possess single-stranded character. Chromatin immunoprecipitation has demonstrated direct association of NME1 with DNA elements in a number of candidate target genes in another melanoma cell line,${ }^{70}$ consistent with function as a canonical transcription factor, and many of those elements possess single-stranded character. Other transcriptional regulators with specificity for single-stranded motifs have been described, such as the single-stranded DNA-binding factors that regulate transcription of the vascular smooth muscle actin promoter. ${ }^{71}$ Nevertheless, a transcriptional coregulatory function (ie, non-DNA binding) is yet to be excluded for NME1, nor have post-transcriptional regulatory mechanisms.

Potential cancer-regulating functions have been ascribed to many of the NME1-regulated genes identified in our study, but the direction of regulation by NME1 was sometimes opposite of expected. For example, the NME1-upregulated genes EREG, CXCL11, NETO2, and XAGE1A have functional properties that are potentially cancer-driving in nature and their expression has been associated in some instances with cancer and malignant progression. ${ }^{42,72,73}$ These discrepancies could be explained by non-canonical functions in the setting of melanoma, tumor cell heterogeneity, or NME1-mediated regulation of expression of these proteins (eg, translation initiation and protein stability) more concordant with their known cancer-relevant functions.

In a prior study conducted in the breast carcinoma cell line MDA-MB-435, microarray analysis also identified a large number of genes whose intracellular concentrations are regulated by NME1. Of particular interest were a number of RNA-processing factors, such as GEMIN5, BOP1, ACIN1, $P A B P$, and $H N R N P A 2 B,{ }^{74}$ suggesting that NME1 may regulate gene expression via impacts on RNA splicing, stability, and other post-transcriptional events. Interestingly, only one gene regulated by NME1 in our melanoma-focused study, NETO2, was also shown to be regulated in the breast carcinoma cell analysis. ${ }^{14}$ Moreover, while NME1 upregulated expression of NETO2 RNA in both the WM793 and M14 melanoma cell lines, NETO2 was downregulated by NME1 in MDA-MDB-435 breast carcinoma cells. As discussed above, the discrepancy in regulation of NETO2 expression between melanoma and breast carcinoma-derived cell lines could be secondary to cell-specific differences in NETO2 function, tumor cell heterogeneity, and/or post-transcriptional processes. Also of note in our analysis was a prevalence of NME1regulated cell surface receptors, consistent with prior studies conducted across a spectrum of cancer cell lines. ${ }^{8,59,74}$

This study has identified a number of NME1-regulated transcripts that may have applications in diagnosis and prognosis for melanoma. Adapting this approach to other known MSGs would seem to hold potential for providing a wealth of novel prognostic markers and therapeutic targets for metastatic disease across a spectrum of human cancers. Whereas expression of NME1 RNA has been shown to be associated with clinical outcome in melanoma, ${ }^{8}$ and the initial identification of NME1 as a metastasis suppressor evolved from an observation of reduced NME1 RNA expression in metastatic melanoma cells, ${ }^{7}$ expression of NME1 RNA itself has not proven to be a robust marker in melanoma or other cancers. This may well be due to the fact that reduced NME1 protein expression in metastatic melanoma cells is often not the result of reduced steady-state concentrations of the cognate NME1 RNA but instead caused by protein destabilization and lysosomal degradation. ${ }^{59}$ Our study provides proof-of-principle that NME1-regulated transcripts and their encoded proteins both individually and collectively hold promise as molecular markers for identifying melanoma subtypes, obtaining more reliable prognoses, and providing personalized medicine for optimal delivery of therapy. In addition, they represent promising molecular targets for development of novel therapies to manage melanoma in its advanced and lethal forms.

Supplementary Information accompanies the paper on the Laboratory Investigation website (http://www.laboratoryinvestigation.org)

\section{ACKNOWLEDGMENTS}

This work was supported by grants from the National Instititues of Health/ National Cancer Institute (CA83237, CA159871, and CA159871-S1).

\section{DISCLOSURE/CONFLICT OF INTEREST}

The authors declare no conflict of interest.

1. Shevde LA, Welch DR. Metastasis suppressor pathways-an evolving paradigm. Cancer Lett 2003;198:1-20.

2. DePeralta DK, Boland GM. Melanoma: advances in targeted therapy and molecular markers. Ann Surg Oncol 2015;22:3451-3458.

3. Tsai KK, Daud Al. The role of anti-PD-1/PD-L1 agents in melanoma: progress to date. Drugs 2015;75:563-575. 
4. Hurst DR, Welch DR. Metastasis suppressor genes: at the interface between the environment and tumor cell growth. Int Rev Cell Mol Biol 2011;286:107-180.

5. Smith SC, Theodorescu D. Learning therapeutic lessons from metastasis suppressor proteins. Nat Rev Cancer 2009;9:253-264.

6. Jayawardana K, Schramm SJ, Tembe V, et al. Identification, review, and systematic cross-validation of microRNA prognostic signatures in metastatic melanoma. J Invest Dermatol 2016;136:245-254.

7. Steeg PS, Bevilacqua G, Kopper L, et al. Evidence for a novel gene associated with low tumor metastatic potential. J Natl Cancer Inst 1988;80:200-204.

8. Winnepenninckx V, Lazar V, Michiels S, et al. Gene expression profiling of primary cutaneous melanoma and clinical outcome. J Natl Cancer Inst 2006;98:472-482.

9. Hartsough MT, Steeg PS. Nm23/nucleoside diphosphate kinase in human cancers. J Bioenerg Biomembr 2000;32:301-308.

10. MacDonald NJ, De La Rosa A, Steeg PS. The potential roles of $n m 23$ in cancer metastasis and cellular differentiation. Eur J Cancer 1995;31A: 1096-1100.

11. Zhang $Q$, McCorkle JR, Novak M, et al. Metastasis suppressor function of NM23-H1 requires its 3';-5' exonuclease activity. Int J Cancer 2011;128:40-50.

12. Jarrett SG, Novak M, Harris N, et al. NM23 deficiency promotes metastasis in a UV radiation-induced mouse model of human melanoma. Clin Exp Metastasis 2013;30:25-36.

13. Snider NT, Altshuler PJ, Omary MB. Modulation of cytoskeletal dynamics by mammalian nucleoside diphosphate kinase (NDPK) proteins. Naunyn Schmiedebergs Arch Pharmacol 2015;388:189-197.

14. Horak CE, Lee JH, Elkahloun AG, et al. Nm23-H1 suppresses tumor cell motility by down-regulating the lysophosphatidic acid receptor EDG2. Cancer Res 2007;67:7238-7246.

15. McCorkle JR, Leonard MK, Kraner SD, et al. The metastasis suppressor NME1 regulates expression of genes linked to metastasis and patient outcome in melanoma and breast carcinoma. Cancer Genomics Proteomics 2014;11:175-194.

16. Boissan $M$, Dabernat $S$, Peuchant $E$, et al. The mammalian Nm23/NDPK family: from metastasis control to cilia movement. Mol Cell Biochem 2009;329:51-62.

17. Agarwal RP, Robinson B, Parks RE. Nucleoside diphosphokinase from erythrocytes. Methods Enzymol 1978;51:376-386.

18. Crawford RM, Treharne KJ, Arnaud-Dabernat S, et al. Understanding the molecular basis of the interaction between NDPK-A and AMPK alpha 1. Mol Cell Biol 2006;26:5921-5931.

19. Lee HY, Lee $\mathrm{H}$. Inhibitory activity of $\mathrm{nm} 23-\mathrm{H} 1$ on invasion and colonization of human prostate carcinoma cells is not mediated by its NDP kinase activity. Cancer Lett 1999;145:93-99.

20. Boissan M, Montagnac G, Shen $Q$, et al. Membrane trafficking. Nucleoside diphosphate kinases fuel dynamin superfamily proteins with GTP for membrane remodeling. Science 2014;344:1510-1515.

21. Postel EH, Berberich SJ, Flint SJ, et al. Human c-myc transcription factor PuF identified as nm23-H2 nucleoside diphosphate kinase, a candidate suppressor of tumor metastasis. Science 1993:261:478-480.

22. Ma D, Xing Z, Liu B, et al. NM23-H1 cleaves and represses transcriptional activity of nuclease-hypersensitive elements in the PDGF-A promoter. J Biol Chem 2002;277:1560-1567.

23. Postel E, Berberich SJ, Rooney JW, et al. Human NM23/nucleoside diphosphate kinase regulates gene expression through DNA binding to nuclease-hypersensitive elements. J Bioenerg Biomembr 2000;32: 277-284.

24. Yang $M$, Jarrett SG, Craven R, et al. YNK1, the yeast homolog of human metastasis suppressor NM23, is required for repair of UV radiation- and etoposide-induced DNA damage. Mutat Res 2009;660:74-78.

25. Jarrett SG, Novak M, Dabernat S, et al. Metastasis suppressor NM23-H1 promotes repair of UV-induced DNA damage and suppresses UVinduced melanomagenesis. Cancer Res 2012;72:133-143.

26. Ma D, McCorkle JR, Kaetzel DM. The metastasis suppressor NM23-H1 possesses 3'-5' exonuclease activity. J Biol Chem 2004;279: 18073-18084.

27. Postel EH, Abramczyk BA, Gursky SK, et al. Structure-based mutational and functional analysis identify human NM23-H2 as a multifunctional enzyme. Biochemistry 2002;41:6330-6337.

28. Freije JM, Blay P, MacDonald NJ, et al. Site-directed mutation of Nm23 $\mathrm{H} 1$. Mutations lacking motility suppressive capacity upon transfection are deficient in histidine-dependent protein phosphotransferase pathways in vitro. J Biol Chem 1997;272:5525-5532.

29. MacDonald NJ, Freije JM, Stracke ML, et al. Site-directed mutagenesis of $\mathrm{nm} 23-\mathrm{H} 1$. Mutation of proline 96 or serine 120 abrogates its motility inhibitory activity upon transfection into human breast carcinoma cells. J Biol Chem 1996;271:25107-25116.

30. Ganguly SS, Fiore LS, Sims JT, et al. c-Abl and Arg are activated in human primary melanomas, promote melanoma cell invasion via distinct pathways, and drive metastatic progression. Oncogene 2012;31:1804-1816.

31. Irizarry RA, Hobbs B, Collin F, et al. Exploration, normalization, and summaries of high density oligonucleotide array probe level data. Biostatistics 2003:4:249-264.

32. Huang da W, Sherman BT, et al. Systematic and integrative analysis of large gene lists using DAVID bioinformatics resources. Nat Protoc 2009;4:44-57.

33. Huang da W, Sherman BT, et al. Bioinformatics enrichment tools: paths toward the comprehensive functional analysis of large gene lists. Nucleic Acids Res 2009;37:1-13.

34. Parnell LD, Lindenbaum $\mathrm{P}$, Shameer $\mathrm{K}$, et al. BioStar: an online question $\&$ answer resource for the bioinformatics community. PLoS Comput Biol 2011;7:e1002216.

35. Alexaki VI, Javelaud D, Van Kempen LC, et al. GLI2-mediated melanoma invasion and metastasis. J Natl Cancer Inst 2010;102:1148-1159.

36. Steder M, Alla V, Meier C, et al. DNp73 exerts function in metastasis initiation by disconnecting the inhibitory role of EPLIN on IGF1R-AKT/ STAT3 signaling. Cancer Cell 2013;24:512-527.

37. Sonoda I, Imoto I, Inoue J, et al. Frequent silencing of low density lipoprotein receptor-related protein 1B (LRP1B) expression by genetic and epigenetic mechanisms in esophageal squamous cell carcinoma. Cancer Res 2004;64:3741-3747.

38. Prazeres $\mathrm{H}$, Torres J, Rodrigues $\mathrm{F}$, et al. Chromosomal, epigenetic and microRNA-mediated inactivation of LRP1B, a modulator of the extracellular environment of thyroid cancer cells. Oncogene 2011;30: 1302-1317.

39. Nikolaev SI, Rimoldi D, Iseli C, et al. Exome sequencing identifies recurrent somatic MAP2K1 and MAP2K2 mutations in melanoma. Nat Genet 2012;44:133-139.

40. Pencheva $\mathrm{N}$, Tran $\mathrm{H}$, Buss $\mathrm{C}$, et al. Convergent multi-miRNA targeting of ApoE drives LRP1/LRP8-dependent melanoma metastasis and angiogenesis. Cell 2012;151:1068-1082.

41. Singh AK, Arya RK, Trivedi AK, et al. Chemokine receptor trio: CXCR3, CXCR4 and CXCR7 crosstalk via CXCL11 and CXCL12. Cytokine Growth Factor Rev 2013:24:41-49.

42. Hensbergen PJ, Wijnands PG, Schreurs MW, et al. The CXCR3 targeting chemokine CXCL11 has potent antitumor activity in vivo involving attraction of CD8+ T lymphocytes but not inhibition of angiogenesis. J Immunother 2005:28:343-351.

43. Chu Y, Yang X, Xu W, et al. In situ expression of IFN-gamma-inducible T cell alpha chemoattractant in breast cancer mounts an enhanced specific anti-tumor immunity which leads to tumor regression. Cancer Immunol Immunother 2007;56:1539-1549.

44. Chheda ZS, Sharma RK, Jala VR, et al. Chemoattractant receptors BLT1 and CXCR3 regulate antitumor immunity by facilitating CD8+ T cell migration into tumors. J Immunol 2016;197:2016-2026.

45. Mamczur P, Gamian A, Kolodziej J, et al. Nuclear localization of aldolase A correlates with cell proliferation. Biochim Biophys Acta 2013;1833: 2812-2822.

46. Ritterson LC, Tolan DR. Targeting of several glycolytic enzymes using RNA interference reveals aldolase affects cancer cell proliferation through a non-glycolytic mechanism. J Biol Chem 2012;287: 42554-42563.

47. Ritterson LC, Tolan DR. Aldolase sequesters WASP and affects WASP/Arp2/3-stimulated actin dynamics. J Cell Biochem 2013;114: 1928-1939.

48. Merkulova M, Hurtado-Lorenzo A, Hosokawa $\mathrm{H}$, et al. Aldolase directly interacts with ARNO and modulates cell morphology and acidic vesicle distribution. Am J Physiol Cell Physiol 2011;300:C1442-C1455.

49. Li YJ, Huang TH, Hsiao M, et al. Suppression of fructose-bisphosphate aldolase $\mathrm{C}$ expression as a predictor of advanced oral squamous cell carcinoma. Head Neck 2016;38(Suppl 1):E1075-E1085.

50. Cunha ES, Kawahara R, Kadowaki MK, et al. Melanogenesis stimulation in B16-F10 melanoma cells induces cell cycle alterations, increased 
ROS levels and a differential expression of proteins as revealed by proteomic analysis. Exp Cell Res 2012;318:1913-1925.

51. Chambers D, Wilson LJ, Alfonsi F, et al. Rhombomere-specific analysis reveals the repertoire of genetic cues expressed across the developing hindbrain. Neural Dev 2009;4:6.

52. Barriga $\mathrm{EH}$, Maxwell $\mathrm{PH}$, Reyes $\mathrm{AE}$, et al. The hypoxia factor Hif-1alpha controls neural crest chemotaxis and epithelial to mesenchymal transition. J Cell Biol 2013;201:759-776.

53. Zendman AJ, van Kraats AA, den Hollander Al, et al. Characterization of XAGE-1b, a short major transcript of cancer/testis-associated gene XAGE-1, induced in melanoma metastasis. Int J Cancer 2002;97: 195-204.

54. Oparina NI, Sadritdinova AF, Snezhkina AV, et al. [Increase in NETO2 gene expression is a potential molecular genetic marker in renal and lung cancers]. Genetika 2012;48:599-607.

55. Calicchio ML, Collins T, Kozakewich HP. Identification of signaling systems in proliferating and involuting phase infantile hemangiomas by genomewide transcriptional profiling. Am J Pathol 2009;174:1638-1649.

56. Kung $C P$, Khaku $S$, Jennis $M$, et al. Identification of TRIML2, a novel p53 target, that enhances p53 SUMOylation and regulates the transactivation of proapoptotic genes. Mol Cancer Res 2015;13:250-262.

57. Paolino $\mathrm{M}$, Choidas A, Wallner $\mathrm{S}$, et al. The E3 ligase Cbl-b and TAM receptors regulate cancer metastasis via natural killer cells. Nature 2014:507:508-512.

58. Dadakhujaev S, Salazar-Arcila C, Netherton SJ, et al. A novel role for the SUMO E3 ligase PIAS1 in cancer metastasis. Oncoscience 2014;1: 229-240.

59. Fiore LS, Ganguly SS, Sledziona J, et al. c-Abl and Arg induce cathepsinmediated lysosomal degradation of the NM23-H1 metastasis suppressor in invasive cancer. Oncogene 2014;33:4508-4520.

60. Tang T, Eldabaje R, Yang L. Current status of biological therapies for the treatment of metastatic melanoma. Anticancer Res 2016;36: 3229-3241.

61. Mehta S, Shelling A, Muthukaruppan A, et al. Predictive and prognostic molecular markers for cancer medicine. Ther Adv Med Oncol 2010;2: 125-148.
62. Vinay DS, Ryan EP, Pawelec $G$, et al. Immune evasion in cancer: mechanistic basis and therapeutic strategies. Semin Cancer Biol 2015;35 Suppl:S185-S198.

63. Kitamura T, Qian BZ, Pollard JW. Immune cell promotion of metastasis. Nat Rev Immunol 2015;15:73-86.

64. Shakhova O. Neural crest stem cells in melanoma development. Curr Opin Oncol 2014;26:215-221.

65. Oskarsson T, Batlle E, Massague J. Metastatic stem cells: sources, niches, and vital pathways. Cell Stem Cell 2014;14:306-321.

66. Lee HJ, Wall B, Chen S. G-protein-coupled receptors and melanoma. Pigment Cell Melanoma Res 2008;21:415-428.

67. O'Hayre M, Vazquez-Prado J, Kufareva I, et al. The emerging mutational landscape of $\mathrm{G}$ proteins and $\mathrm{G}$-protein-coupled receptors in cancer. Nat Rev Cancer 2013;13:412-424.

68. Wei X, Walia V, Lin JC, et al. Exome sequencing identifies GRIN2A as frequently mutated in melanoma. Nat Genet 2011;43:442-446.

69. Gelis $L$, Jovancevic N, Veitinger $S$, et al. Functional characterization of the odorant receptor 51E2 in human melanocytes. J Biol Chem 20162011;291:17772-17786.

70. Cervoni L, Egistelli L, Eufemi M, et al. DNA sequences acting as binding sites for NM23/NDPK proteins in melanoma M14 cells. J Cell Biochem 2006;98:421-428.

71. Sun S, Stoflet ES, Cogan JG, et al. Negative regulation of the vascular smooth muscle alpha-actin gene in fibroblasts and myoblasts: disruption of enhancer function by sequence-specific singlestranded-DNA-binding proteins. Mol Cell Biol 1995;15:2429-2436.

72. Zhang J, Iwanaga K, Choi KC, et al. Intratumoral epiregulin is a marker of advanced disease in non-small cell lung cancer patients and confers invasive properties on EGFR-mutant cells. Cancer Prev Res 2008;1: 201-207.

73. Hu K, Li SL, Gan YH, et al. Epiregulin promotes migration and invasion of salivary adenoid cystic carcinoma cell line SACC-83 through activation of ERK and Akt. Oral Oncol 2009;45:156-163.

74. Lee JH, Horak CE, Khanna C, et al. Alterations in Gemin5 expression contribute to alternative mRNA splicing patterns and tumor cell motility. Cancer Res 2008;68:639-644. 